\title{
LOCAL STRUCTURE AND MOLECULAR CORRELATIONS IN LIQUID NAPHTHALENE
}

\author{
Z. BoChyŃSKI AND L. DEJNEKA \\ Non-Crystalline Materials Division, Institute of Physics, Adam Mickiewicz University \\ Grunwaldzka 6, 60-780 Poznań 2, Poland
}

(Received June 4, 1996; revised version April 8, 1997;

in final form November 12, 1997)

\begin{abstract}
$\mathrm{X}$-ray diffraction studies of liquid naphthalene and, for the sake of comparison, of liquid benzene and diphenyl were performed. The results permitted the determination of the intermolecular distances and the packing coefficient. A comparison of the structures of liquid naphthalene, benzene, and diphenyl shows that in benzene and diphenyl 3 coordination spheres of molecular ordering can be distinguished in the range up to $18 \AA$, while in naphthalene only two in the range up to $15 \AA$. Analysis of the space packing in (benzene, naphthalene, and diphenyl) all liquids studied has proved its substantial increase with increasing volume of their molecules.
\end{abstract}

PACS numbers: $61.25 . \mathrm{Em}$

\section{Introduction}

The subject of the studies reported in this work is naphthalene. For the sake of comparison similar studies were performed for benzene and diphenyl. The molecules of naphthalene and diphenyl include in their structure a well known molecule of benzene.

Benzene was the first molecular liquid to have its structure resolved. The first results on this liquid were published in 1915 [1]. Numerous X-ray studies confirmed its planar hexagonal structure [2-5]. Diphenyl has also been the subject of X-ray studies [6-8] and its structural parameters and local ordering degree, also in coordination spheres of higher orders, were determined [8]. The results reported in this work are in good agreement with the literature provided data.

The first attempt to study naphthalene in the liquid phase by X-ray was undertaken as early as in 1930 [9], however, the data gathered so far are fragmentary and scarce.

This work reports the first studies of liquid naphthalene performed by the counter method for the range of the angular measurements intensity extended to the value of $\vartheta=60^{\circ}$. Also for the first time the range of distances in the calcula- 
tions of the radial distribution function (RDF) for liquid naphthalene was extended to include the intermolecular distances of over $20 \AA$. The calculations by similar Fourier transform methods over a similarly large range of intermolecular distances had been already done earlier for benzene and diphenyl [8]. For naphthalene and diphenyl, the distributions of scattered radiation intensity were expressed as a function of the wave vector $S$ and the corrected Bragg equation $R$, and compared with structural data for benzene. Moreover, a comparison of the total and differential radial distribution functions of electron density for these three liquids was made.

\section{Experimental}

The structure of the molecular liquids: benzene, naphthalene, and diphenyl was studied by diffraction of strictly monochromatic X-ray radiation. The measurements were performed on a universal X-ray diffractometer HZG 4 with a lamp with a copper anode working at the optimum voltage of $U=40 \mathrm{kV}$ and current intensity $I=30 \mathrm{~mA}$, using a proportional counter. The monochromatisation of the $K_{\alpha}$-radiation of $\lambda=1.5418 \AA$ was ensured by a special graphite monochromator constructed in our laboratory.

Measurements were carried out by the transmission method with the preparation mounted symmetrically with respect to the incident and reflected beams. The liquid studied was placed in a plane-parallel cell of adjustable thickness and with windows of thin mica layer. The measuring chamber was dynamically thermostated within the range from $20^{\circ} \mathrm{C}$ to $200^{\circ} \mathrm{C}$.

\section{TABLE I}

Physical and chemical parameters of liquid diphenyl, naphthalene, and benzene [8].

\begin{tabular}{l|c|c|c}
\hline \hline \multicolumn{1}{c|}{$\begin{array}{c}\text { Physical and chemical } \\
\text { parameters }\end{array}$} & \multicolumn{3}{|c}{ Compounds } \\
\cline { 2 - 4 } & Diphenyl & Naphthalene & Benzene \\
\hline Electron density $\rho_{0}\left[\mathrm{el} . / \AA^{3}\right]$ & 0.315 & 0.308 & 0.283 \\
Melting point & 70 & 80.2 & 5 \\
Temperature of measurements $\left[{ }^{\circ} \mathrm{C}\right]$ & 75 & 95 & 20
\end{tabular}

The functions of the angular distribution of scattered radiation were determined at certain temperatures given in Table I for angles from the range $6-120^{\circ}$ read out with an accuracy of $\Delta \vartheta=0.005^{\circ}$. The course of the intensity changes as a function of $\vartheta$ was corrected for the background, absorption, polarization, and incoherence of radiation. These functions were then normalized by expressing them in the electronic units. The intensity in the electronic units was presented as a function of $S$ defined by the formula $S=(4 \pi \sin \vartheta) / \lambda$, where $\vartheta$ is the Bragg angle, $\lambda$ is the wavelength of $\mathrm{X}$-ray radiation [10-12].

Moreover, the measured intensity of the scattered radiation was also displayed as a function of $R$ defined as follows: $R=1.25 \times 2 \pi / S$ [13]. 
A complete Fourier analysis of these functions has been performed on the basis of the Warren-Krutter-Morningstar equation [11]:

$$
4 \pi r^{2} \sum \bar{K}_{\mathrm{m}} \rho_{\mathrm{m}}(r)=4 \pi r^{2} \rho_{0} \sum \bar{K}_{\mathrm{m}}+\frac{2 r}{\pi} \int_{0}^{\infty} S i(S) \sin (S r) \mathrm{d} S,
$$

where $\rho_{\mathrm{m}}(r)$ is the atomic density and $r$ - the interatomic distance, $\rho_{0}$ is the mean electron density calculated from the equation: $\rho_{0}=d \sum_{m=1}^{t} Z_{m} / M$, where $d$ is the macroscopic density, $M-$ molecular mass, $Z_{m}$ - the number of electrons in a molecule and $t$ - the number of atoms in a molecule.

The effective number of the scattering electrons in a molecule is given by

$$
K_{\mathrm{m}}(S)=f_{\mathrm{m}}(S) / f_{\mathrm{e}}(S),
$$

where $f_{\mathrm{m}}(S)$ is the atomic factor and $f_{\mathrm{e}}(S)$ is the mean value of the atomic factor of a molecule per a single electron

$$
f_{\mathrm{e}}=\sum f_{\mathrm{m}}(S) / \sum Z_{m}
$$

The mean effective number of the scattering electrons $K_{\mathrm{m}}$ is defined as

$$
\bar{K}_{\mathrm{m}}=\frac{1}{S} \int_{0}^{S} K_{\mathrm{m}}(S) \mathrm{d} S
$$

The parameter $i(S)$ occurring in the integrand in the Warren-Krutter-Morningstar equation is defined as

$$
i(S)=\left[\bar{I}(S) / N-\sum f_{\mathrm{m}}{ }^{2}(S)\right] / f_{\mathrm{e}}{ }^{2}(S),
$$

where $N$ is the number of molecules in the preparation studied.

The total as well as differential functions of radial distribution of intensity were calculated by the Simpson integration method for $0 \leq r \leq 20$ at every $0.05 \AA$, based on the parabolic interpolation. The calculation of the radial distribution functions was performed with the use of the computer program ZMIANA OO written by Kowalski and Drozdowski $[8,14]$.

\section{Conclusions}

A comparison of the results of structural analyses of benzene, naphthalene, and diphenyl was made. The data characterising physical and chemical properties of these compounds are collected in Tables I and II.

Molecular volumes calculated from the increments grow in the sequence: benzene, naphthalene, diphenyl (Table II).

Increase in the mean effective number of scattering electrons in a molecule in the sequence: benzene, naphthalene, and diphenyl, is also manifested as growing area under the curve of the scattered radiation intensity (Fig. 1).

A comparison of the character of $\bar{I}(S)$ functions of these compounds reveals a shift of their maxima towards decreasing values of $S$ (Table III).

The same was the sequence of growing electron density manifested as increasing area under the RDF maxima (Fig. 2).

Significant differences were noted in the character of differential functions obtained for the three compounds (Fig. 3). These functions bring information about the difference between the observed and the average distribution of electron 

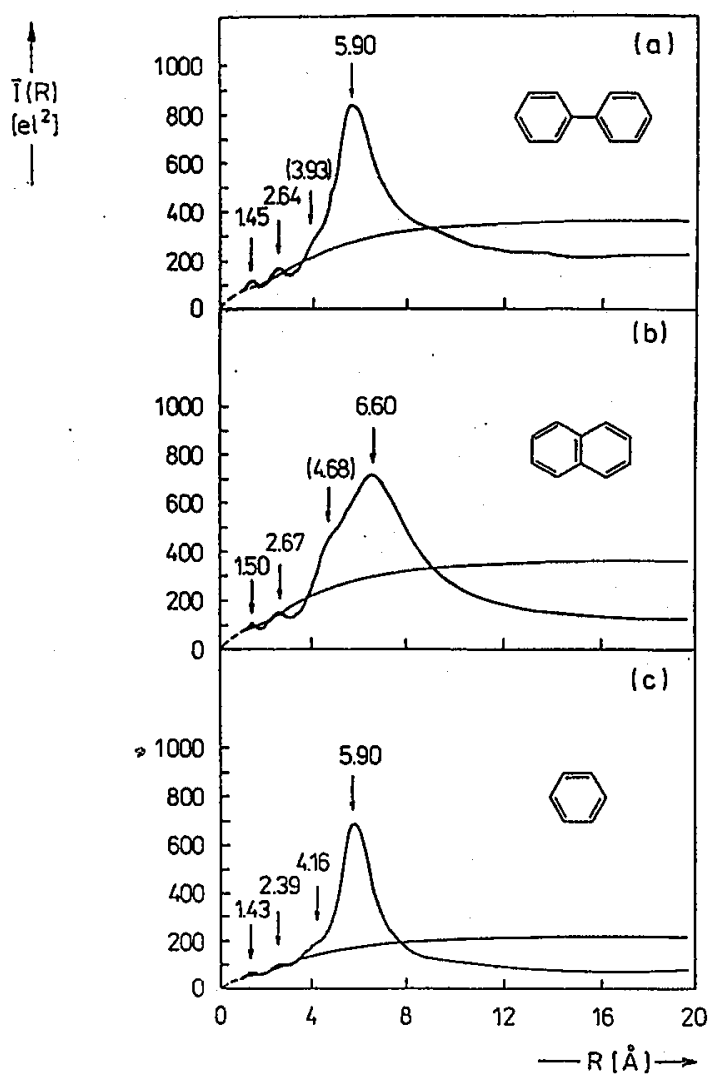

Fig. 1. Intensity distribution of X-radiation scattered in liquid diphenyl (a), naphthalene (b), and benzene (c) expressed as a function of $R$.

TABLE II

Structural parameters of the liquid diphenyl, naphthalene, and benzene [8].

\begin{tabular}{l|c|c|c}
\hline \hline Structural parameters & \multicolumn{3}{|c}{ Compounds } \\
\cline { 2 - 4 } & Diphenyl & Naphthalene & Benzene \\
\hline $\begin{array}{c}\text { Mean effective number of carbon atom } \\
\text { electrons in molecule } \bar{K}_{\mathrm{C}}\end{array}$ & 6.314 & 6.303 & 6.369 \\
$\begin{array}{c}\text { Mean effective number of hydrogen atom } \\
\text { electrons in molecule } \bar{K}_{\mathrm{H}}\end{array}$ & 0.648 & 0.647 & 0.652 \\
$\begin{array}{c}\text { Mean effective number of electrons } \\
\text { in molecule } \sum \bar{K}_{\mathrm{m}}\end{array}$ & 82.248 & 68.206 & 42.126 \\
$\begin{array}{l}\text { Molecular volume } V_{0}\left[\AA^{3}\right] \\
\text { Packing coefficient of molecules } \\
\text { in liquids }\end{array}$ & 163.8 & 134.4 & 88.2 \\
& 0.629 & 0.611 & 0.594
\end{tabular}




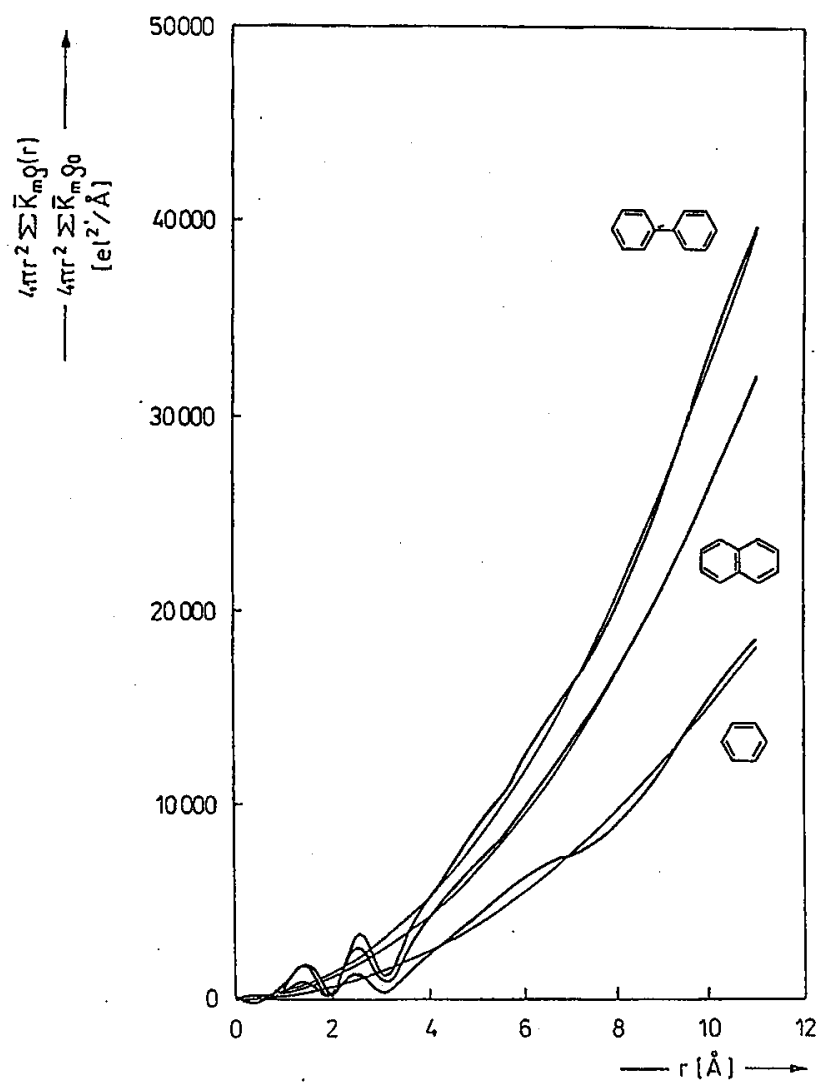

Fig. 2. Total functions of radial distribution of electron density for liquid diphenyl, naphthalene, and benzene.

TABLE III

\begin{tabular}{l|c|c|c} 
Maxima of the $\bar{I}(S)$ function for the liquids studied. \\
\hline \hline Compounds & \multicolumn{3}{|c}{ Maxima of $\bar{I}(S)$} \\
\cline { 2 - 4 } & $S_{1}\left[\AA^{-1}\right]$ & $S_{2}\left[\AA^{-1}\right]$ & $S_{3}\left[\AA^{-1}\right]$ \\
\hline Diphenyl & 1.33 & 2.97 & 5.43 \\
Naphthalene & 1.19 & 2.95 & 5.24 \\
Benzene & 1.33 & 3.29 & 5,47
\end{tabular}

density. A clearly evidenced split of the main maximum of the differential radial distribution function, corresponding to the distance of $6.10 \AA$ for benzene, for the functions of naphthalene and diphenyl implies that the local simple configurations present in benzene become more complex with addition of subsequent molecular segment.

Assuming the rigid and planar conformation of the molecules of the compounds studied, models of their structure were proposed. The probable confor- 


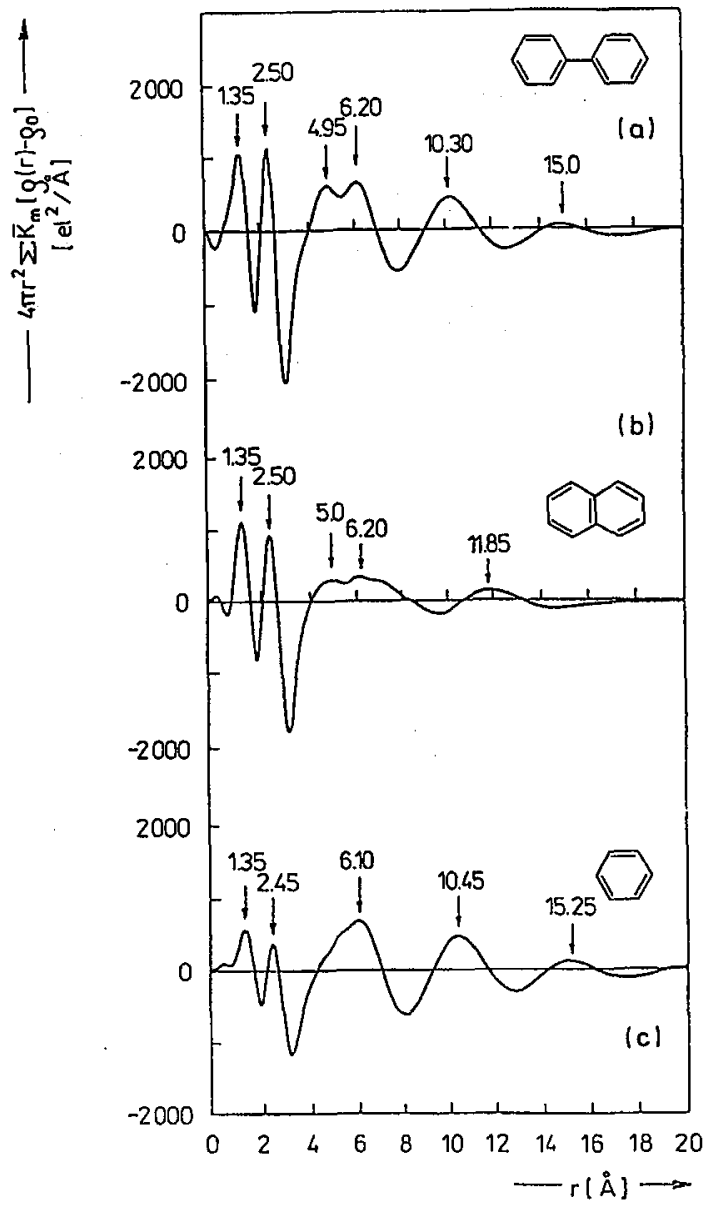

Fig. 3. Differential functions of radial distribution of electron density for liquid diphenyl (a), naphthalene (b), and benzene (c).

mations of these molecules are shown in Fig. 4, where the mean intermolecular distances are marked.

The range of intermolecular distances corresponding to the main maximum of the differential function for benzene covers those characteristic of almost perpendicular arrangement of molecules both in the form of letters $\mathrm{T}$ and $\mathrm{L}$. The maximum of this function at $r=6.10 \AA$ for benzene correspond to the $\mathrm{L}$ type. arrangement (with $\alpha \approx 74^{\circ}$ ), see Fig. 4a, which means that this conformation prevails among the nearest neighbours in benzene.

A clearly marked split of this maximum observed for diphenyl corresponding to $r=4.95 \AA$ and $r=6.20 \AA$, indicates a possibility of the presence of two probable arrangements in the first coordination sphere. The position of the first of the maxima is attributed to the skew conformation of the nearest neighbour molecules at an angle of $\alpha \approx 72^{\circ}$, while the other maximum is interpreted as 
a)

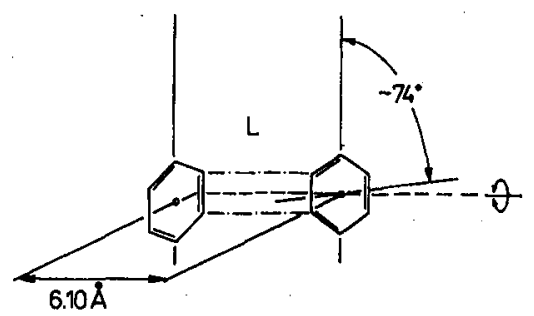

b)

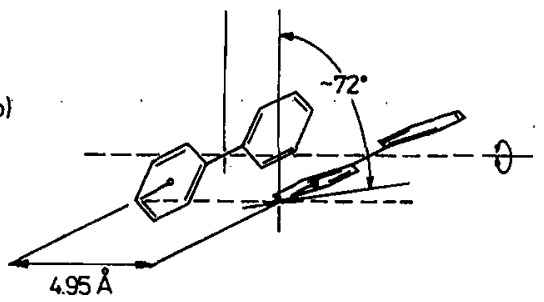

c)

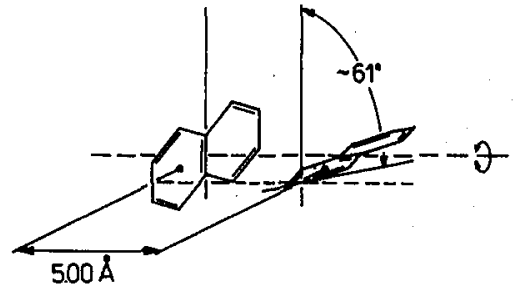

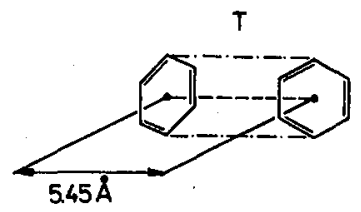
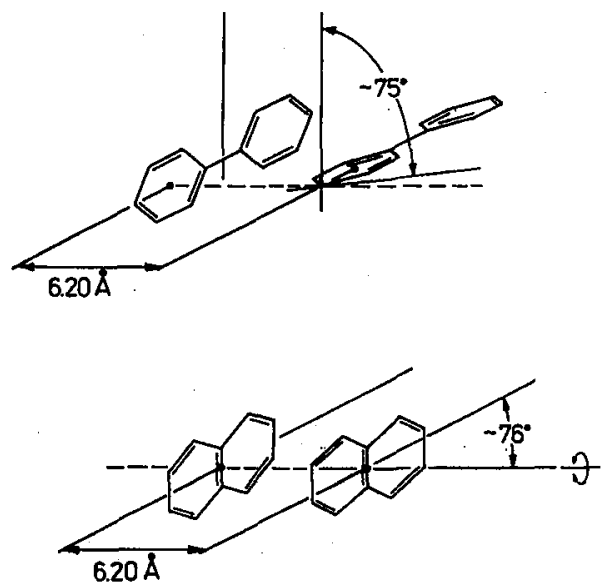

Fig. 4. Models of probable conformations of molecules in benzene (a), diphenyl (b), and naphthalene (c).

\section{TABLE IV}

The radii of coordination spheres in liquid diphenyl, naphthalene, and benzene.

\begin{tabular}{l|c|c|c}
\hline \hline Compounds & \multicolumn{3}{|c}{ Spheres $[\AA]$} \\
\cline { 2 - 4 } & 1st sphere & 2nd sphere & 3rd sphere \\
\hline Diphenyl & $3.2-8.0$ & $8.0-12.7$ & $12.6-17.5$ \\
Naphthalene & $3.2-9.7$ & $9.7-14.7$ & $14.7-20.4^{*}$ \\
Benzene & $3.2-8.1$ & $8.1-12.8$ & $12.8-17.6$ \\
\hline
\end{tabular}

*estimated values

corresponding to the molecular conformation also close to perpendicular, $\alpha \approx 75^{\circ}$, but with translation as follows from the dense packing model (Fig. $4 \mathrm{~b}$ ).

The broad maximum of the differential function for diphenyl does not exclude the perpendicular conformation of molecules for $r=5.1 \AA$ or the perpendicular conformation with translation for $r=6.3 \AA$, however, suggests that these conformations are less probable than the skew one.

The skew conformation is probably assumed because it is more energetically favourable and enables a more favourable packing of the molecules. 
The split of the main maximum of the radial distribution function is also well marked for naphthalene. For this compound the position of the first maximum of the radial distribution function at $r=5.0 \AA$ corresponds to the skew orientation of the nearest neighbours with $\alpha \approx 61^{\circ}$, while the position of the other one corresponds to the almost perpendicular molecular arrangement with $\alpha \approx 76^{\circ}$ (Fig. $4 \mathrm{c}$ ). Also in this case the symmetrical arrangements corresponding to $r=5.50 \AA$ or $r=6.34 \AA$ are less probable. The split of the main maximum of the radial distribution function and the significant broadening of the coordination spheres (Table IV) testify to the fact that molecular conformations of the nearest neighbours in naphthalene are much more complex than in benzene.

With development and growing complexity of molecular conformations when passing from benzene to the other compounds, the packing coefficient significantly increases reaching the maximum value for diphenyl (Table II), and the arrangement of the nearest neighbours becomes more complicate.

The work was performed within the research project No. 201319101 of the the Committee for Scientific Research.

\section{References}

[1] P. Debye, Ann. Phys. 46, 809 (1915).

[2] Z. Bochyński, Ph.D. Thesis, UAM, Poznań 1965.

[3] Z. Bochyński, Acta Phys. Pol. A 34, 557 (1968).

[4] A.H. Narten, J. Chem. Phys. 48, 1630 (1968).

[5] L.J. Lowden, D. Chandler, J. Chem. Phys. 61, 5228 (1974).

[6] M. Bertrandt-Żytkowiak, PTPN, Fizyka Dielektryków 9, 199 (1977).

[7] M. Bertrandt-Żytkowiak, Ph.D. Thesis, UAM, Poznań 1971.

[8] M. Kowalski, Ph.D. Thesis, UAM, Poznan 1992.

[9] J.R. Katz, J. Selman, Phys. Zeit. 34, 834 (1930).

[10] B.E. Warren, Phys. Rev. 44, 969 (1933).

[11] H. Klug, E. Alexander, X-Ray Diffraction Procedures for Polycrystalline and Amorphous Materials, Wiley, New York 1966.

[12] H.N.V. Temperley, J.S. Rowlinson, G.S. Rushbrooke, Physics of Simple Liquids, North-Holland, Amsterdam 1968.

[13] J.T. Randall, The Diffraction of X-Rays and Electrons by Amorphous Solids, Liquids and Gases, Chapman and Hall, London 1934.

[14] H. Drozdowski, Ph.D. Thesis, UAM, Poznań 1994; Acta Phys. Pol. A 88, 1089 (1995); ibid. 89, 547 (1996). 